Gene expression

\title{
scAnnotate: an automated cell type annotation tool for single-cell RNA-sequencing data
}

\author{
Xiangling Ji, Danielle Tsao, Kailun Bai, Min Tsao, and Xuekui Zhang*
}

Department of Mathematics \& Statistics, University of Victoria, Victoria, British Columbia, Canada V8W 2Y2

*To whom correspondence should be addressed.

Associate Editor: $\mathrm{XXXXXXX}$

Received on XXXXX; revised on XXXXX; accepted on XXXXX

\begin{abstract}
Motivation: Single-cell RNA-sequencing (scRNA-seq) technology enables researchers to investigate a genome at the cellular level with unprecedented resolution. An organism consists of a heterogeneous collection of cell types, each of which plays a distinct role in various biological processes. Hence, the first step of scRNA-seq data analysis often is to distinguish cell types so that they can be investigated separately. Researchers have recently developed several automated cell type annotation tools based on supervised machine learning algorithms, requiring neither biological knowledge nor subjective human decisions. Dropout is a crucial characteristic of scRNA-seq data widely used in differential expression analysis. However, existing supervised learning methods for cell annotation do not use the dropout information explicitly. This motivated us to build a novel cell annotation tool that fully utilizes this information.

Results: We present scAnnotate, an automated cell annotation tool based on supervised machine learning algorithms. We use a marginal mixture model to describe both the dropout proportion and the non-dropout expression level distribution of a gene. We develop a marginal model based ensemble learning approach to avoid having to specify and estimate a high-dimensional joint distribution for all genes. First, we build a 'weak' classifier using the mixture model for each gene. Then, we combine 'weak' classifiers of all genes into a single 'strong' classifier to annotate cells. Using 11 real scRNA-seq data, we demonstrate that scAnnotate is competitive against 9 other methods, and that it accurately annotates cells when training and test data are (1) similar, (2) cross-platform, and (3) cross-species.
\end{abstract}

Availability: An R package, scAnnotate, is in preparation and will be publicly available by March 2022.

Contact: Xuekui Zhang, xuekui@uvic.ca

\section{Introduction}

Every biological process in the human body relies on the coaction of numerous cell types, each with their own designated function. Cell identification is thus crucial in studying biological phenomenons and developing medical practices; pathology, for example, hinges on the accuracy of this task. Although standard immunophenotyping methods are widely practiced for cell identification, their heavy reliance on the manual selection of antibodies, markers and fluorochromes renders new and rare cell types particularly difficult to identify (Gown, 2008; Leach et al., 2013). Conversely, newly developed single-cell RNA sequencing (scRNA-seq) technologies (Tang et al., 2009) have heightened the detail with which we can examine cell composition by offering unprecedented resolution of gene expression at the cellular level. The recent surge of available scRNA-seq data allows for increased accuracy in several aspects of genomic data analysis, including cell annotation (Chen et al., 2019; Diaz-Mejia et al., 2019; Artegiani et al., 2017).

Cell-type annotation using scRNA-seq data enables researchers to distinguish various types of cells from heterozygous populations, then to investigate each cell type separately and to learn their interactions. Hence, cell-type annotation is often the first step of scRNA-seq data analysis, which has led to a recent surge of methods developed for this task. Pasquini et al. (2021) discussed 24 scRNA-seq cell-type annotation methods developed in the last five years. The most popular cell-type annotation approach was clustering analysis followed by manual annotation. The most important advantage of such an approach is that it does not require training a model using another 'annotated' scRNA-seq dataset. However, such unsupervised machine learning approaches have a critical issue; namely, they require users to manually label the cell types for each cluster of cells. 
The manual decisions need special biological knowledge and are subjective to researchers' individual opinions, which can be time-consuming and inconsistent. In the last few years, a huge amount of scRNA-seq data was generated and made publicly available. These rich resources made it easie and easier to identify suitable data for training supervised machine learning models to annotate new scRNA-seq data. Recently, many supervised machine learning methods have been developed for cell-type annotation.

Currently, the discriminative classification approach dominates supervised machine learning methods for cell-type annotation. This discriminative classification approach models the distribution of cell type conditional on genomic data. For example, CaSTLe (Lieberman et al. 2018) employs an XGBoost (Chen and Guestrin, 2016) classification model and SingleCellNet (Tan and Cahan, 2019) trains a Random Forest classifier on discriminating gene pairs. CHETAH (de Kanter et al., 2019) and scClassify (Lin et al., 2020) construct hierarchical classification trees and evaluate the correlation of query cells to reference cell types or apply an ensemble of weighted kNN classifiers, respectively. In SingleR (Aran et al., 2019), the Spearman rank correlations of query cells to reference samples are used in an altered kNN classification algorithm. Similarly, scmap (Kiselev et al., 2018) classifies cells by measuring their similarity to either the centroids of reference clusters (scmap-cluster) or by kNN cell annotation (scmap-cell). Finally, scPred (Alquicira-Hernandez et al., 2019) reduces the reference data's dimensionality using PCA and applies a Support Vector Machine model for classification. The common unwanted characteristic of discriminative classification methods is that they do not utilize the distribution of genomic data. However, the distribution of genomic data carries key features of scRNA-seq data, which should be helpful for cell-type annotation. For example, "dropout" is the well-known sparsity issue characterized by the excessive amount of zero counts in scRNA-seq data, arising from technical limitations in detecting moderate or low gene-expression levels in cells of the same type (Hicks et al., 2018). Various imputation methods were developed to remove dropouts from data such as SAVER (Huang et al., 2018), scImpute ( $\mathrm{Li}$ and $\mathrm{Li}, 2018$ ), and DrImpute (Gong et al., 2018). However, imputation could generate false positive signals within the data due to the intrinsic circularity of current scRNA-seq expression recovery practices (Andrews and Hemberg, 2018). Furthermore, the proportion of dropouts can be helpful information for cell annotation. We therefore prefer to utilize this information instead of removing it.

To fully utilize the unique characteristics of scRNA-seq genomic data, we investigate the generative classification approach which models the distribution of genomic data conditional on cell type. Such an approach focuses on distributions of genomic data in different cell types, and annotates cells using Bayesian theorem. To the best of our knowledge, scID (Boufea et al., 2020) is the only cell-type annotation method based on a generative classifier. scID uses Fisher's linear discriminant analysis (LDA) to distinguish the characteristic genes of pre-determined cell clusters. LDA assumes that genomic data follow a multivariate normal distribution, which might over-simplify the complexity of the data. Furthermore, from data with limited sample size, it is hard to precisely estimate numerous parameters in the high-dimensional covariance matrix of the assumed multivariate normal distribution.

In this paper, we propose a novel generative classifier for automated cell-type annotation, scAnnotate. We focus on addressing the two critica challenges of scRNA-seq data as discussed above: the curse of high dimensionality (as discussed in LDA) and explicitly modelling dropout. To address the curse of high dimensionality, we use every gene to make a classifier and consider it as a 'weak' learner, and then use a combiner function to ensemble 'weak' learners built from all genes into a single 'strong' learner for making the final decision. To select a gene's distribution that explicitly models the excessive zero counts in each weak learner, we seq data. The literature of DE analysis is well-established, with many methods that focus on modelling excessive zero counts. For example, Kharchenko et al. (2014) introduced a Bayesian approach to scRNAseq DE analysis in which non-zero counts are modelled using a Negative Binomial distribution and zero counts are modelled with a low-magnitude Poisson process. DEsingle Miao et al. (2018) is another scRNA-seq DE analysis tool that uses the Zero-Inflated Negative Binomial (ZINB) distribution. However, after batch effect removal and other preprocessing, scRNA-seq data are often no longer integers and hence are not suitable for the ZINB model. Furthermore, recent benchmark studies did not show a clear advantage of ZINB in DE analysis of scRNA-seq data (Soneson and Robinson, 2018). We therefore decide to model gene expression levels as a continuous variable. MAST (Finak et al., 2015) joint models the proportion of dropouts and the distribution of non-dropouts using a hurdle regression model, which is one of the most popular DE analysis softwares, and has shown great performance in benchmark study (Soneson and Robinson, 2018). Inspired by MAST, we joint model the proportion of dropout and gene expressions of non-dropouts by a two-component mixture model. We tried various distributions to model the non-dropout component in the mixture model, and empirically found that the lognormal distribution works best for most of the data that we explored. In the Discussion, we also discuss two alternative distributions implemented in our software that are useful in particular situations. In the rest of this paper, we will introduce the details of the scAnnotate method and use real scRNA-seq datasets to compare its performance of classification with nine other scRNA-seq annotation methods based on supervised machine learning algorithms.

\section{Materials and methods}

We introduce scAnnotate, an automated cell type annotation tool. scAnnotate is entirely data-driven, which requires training data to learn the classifier, but not biological knowledge to make subjective decisions. It consists of three steps: preprocessing training and test data, model fitting on training data, and cell classification on test data. The classification model in the last step uses an ensemble machine learning approach involving many weak learners and a combiner function to integrate outputs of the weak learners into a single strong learner. Each weak learner is a classifier based on a mixture model for the expression level of one gene. The combiner is a weighted average of all weak learners' outputs. The weights can be either learned from training data when the sample size is big enough, or pre-specified as equal weights in small sample applications. scAnnotate handles big data and small data differently. An illustration of its workflow is shown in Figure 1 (small data) and Figure 2 (large data). Details of each element of scAnnotate will be discussed in the rest of this section.

\subsection{Batch effects removal}

When building a supervised machine learning model for cell-type annotation, batch effects often create differences between the training and testing data. We therefore believe that removing batch effects will make the model learned from training data more suitable for annotating cells in test data. For example, scPred (Alquicira-Hernandez et al., 2019) has batch effect removal as a built-in optional step. Following this idea, we suggest using batch effect removal as a data preprocessing step unless users strongly believe their training data and test data are similar enough to each other. scAnnotate removes batch effects using the Seurat package (Hao et al., 2021) for big data (with a mean of 600 observations per cell type and no less than 20 cells for any given type) or the Harmony package (Korsunsky et al., 2018) for small data. Both methods are recommended by (Tran et al., 2020) due to their consistently good performances and 


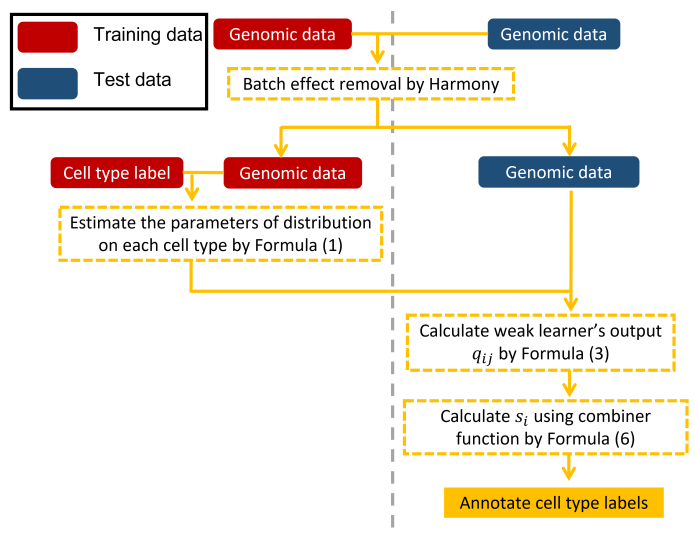

Fig. 1. Workflow of scAnnotate on small sample datasets (with a mean of less than 600 observations per cell type). The gray vertical dashed line separates training data (left) and test data (right) information.

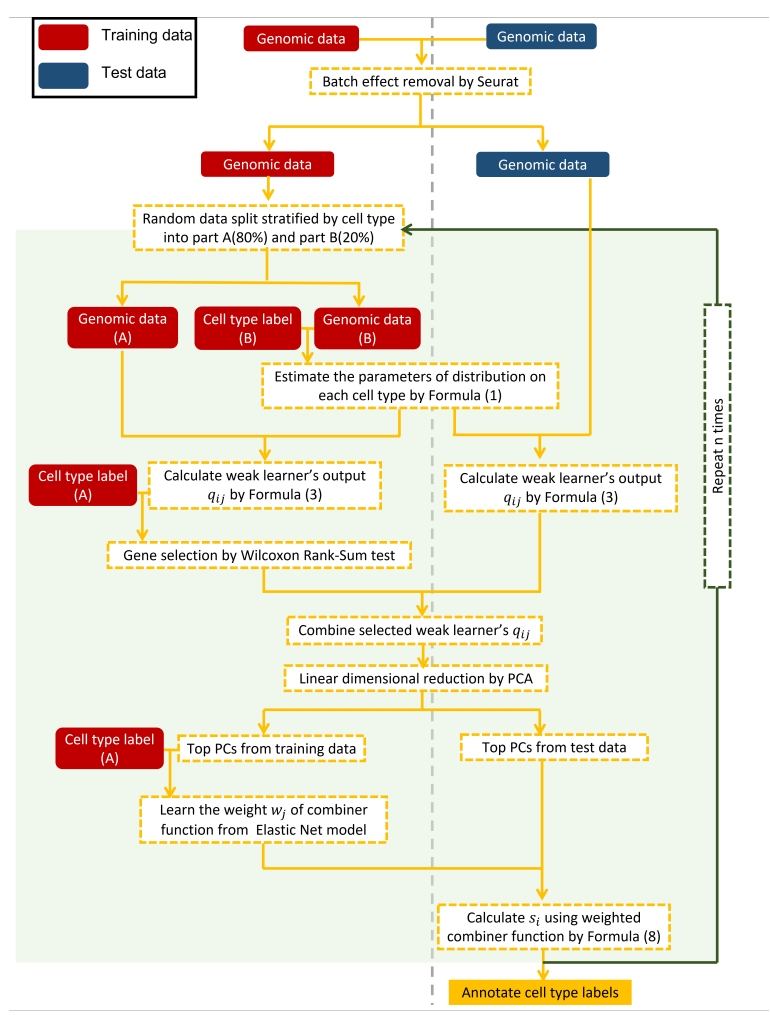

Fig. 2. Workflow of scAnnotate on large sample datasets (with a mean of 600 observation per cell type and no less than 20 cells for any given type). The gray vertical dashed line separates training data (left) and test data (right) information.

comparatively low runtimes. The output of batch effect removal will be used as input for the classification model discussed next.

\subsection{Mixture model for the expression level of a given gene in a fixed cell type}

For the $j$ th (selected) gene in the type- $i$ cell, we propose a mixture mode $F_{i j}$ for its expression level

$$
F_{i j}=p_{i j} F^{0}+\left(1-p_{i j}\right) F_{i j}^{+}, \quad i=1,2, \ldots, n_{t}, j=1,2, \ldots, n_{g},
$$

where $F^{0}$ is the degenerated distribution at $0, F_{i j}^{+}$is a distribution supported on $(0, \infty)$, and $n_{t}$ and $n_{g}$ are, respectively, the total number of cell types and the total number of genes selected for use in classification The $p_{i j}$ and $\left(1-p_{i j}\right)$ are the mixing proportions for $F^{0}$ and $F_{i j}^{+}$, respectively. Model (1) includes commonly used zero-inflated models such as the zero-inflated Poisson model as special cases, but it offers more flexibility than such zero-inflated models as it models the proportion of zeros and the distribution of the positive expression levels $F_{i j}^{+}$separately. In particular, under (1), all distributions supported on $(0, \infty)$ or a subset of $(0, \infty)$ may be used to model $F_{i j}^{+}$. In situations where no good parametric models for $F_{i j}^{+}$are available, we may also specify $F_{i j}^{+}$nonparametrically.

\subsection{Mixture model for the expression level of a given gene,}

\section{its estimation and prior specification}

Let $\pi_{i}$ be the prior probability that a randomly selected cell is of type- $i$ where $\pi_{1}+\pi_{2}+\cdots+\pi_{n_{t}}=1$. The (prior) distribution of the expression level of the $j$ th gene of this cell is the following mixture distribution

$$
F_{j}=\pi_{1} F_{1 j}+\pi_{2} F_{2 j}+\cdots+\pi_{n_{t}} F_{n_{t}, j}
$$

where the $F_{i j}$ are defined in (1). We estimate $F_{i j}$ and $F_{j}$ using training data as follows. Suppose the training data contains $n_{i}$ independent type$i$ cells. Then, there are $n_{i}$ independent observations for the $j$ th gene in type- $i$ cells. Let $k_{i j}^{0}$ be the number of zeros among these $n_{i}$ observations and $k_{i j}^{+}$be the number of positive observations so that $n_{i}=k_{i j}^{0}+k_{i j}^{+}$. Then, we may estimate the mixing proportions $p_{i j}$ in (1) with

$$
\hat{p}_{i j}=k_{i j}^{0} / n_{i}, \quad i=1,2, \ldots, n_{t}, j=1,2, \ldots, n_{g} .
$$

To estimate parameters of the distribution $F_{i j}^{+}$, we use the $k_{i j}^{+}$positive observations. For example, if $F_{i j}^{+}$is assumed to be a lognormal distribution, then we can find the maximum likelihood estimates for its parameters using the $k_{i j}^{+}$positive observations.

The prior probabilities $\pi_{i}$ depend on the application at hand. In the absence of information for determining these probabilities, we recommend the uniform prior $\pi_{i}=1 / n_{t}$. We have also used the observed proportions $\pi_{i}=n_{i} / \sum_{k=1}^{n_{t}} n_{k}$ which is reasonable when the training sample is a random sample from the population of cells of all types.

\subsection{Weak learner based on mixture model of a single gene}

To classify a future cell of unknown type into one of the $n_{t}$ types with its $n_{g}$ gene expression data, we first use the $n_{g}$ genes one at a time to perform the classification. This leads to $n_{g}$ weak learners.

Let $X_{j}$ be the expression level of the $j$ th gene of the cell. Then, since the type of the cell is unknown, $X_{j} \sim F_{j}$ in (2). Let $x_{j}$ be the observed value of $X_{j}$. The posterior probability that the cell is of type- $i$ is

$$
q_{i j}=P\left(\text { type }-i \mid X_{j}=x_{j}\right)=\frac{P\left(X_{j}=x_{j} \mid \text { type }-i\right) P(\text { type }-i)}{P\left(X_{j}=x_{j}\right)}
$$

for $i=1,2, \ldots, n_{t}$, which can be computed by using the estimated $F_{i j}$ and $F_{j}$. Specifically, for $x_{j}=0$, we have

$$
q_{i j}=P\left(\text { type }-i \mid X_{j}=0\right)=\frac{\pi_{i} \hat{p}_{i j}}{\pi_{1} \hat{p}_{1 j}+\pi_{2} \hat{p}_{2 j}+\cdots+\pi_{n_{t}} \hat{p}_{n_{t} j}},
$$

which is the probability that the observed zero comes from a type- $i$ cell. For $x_{j}>0$, we have

$$
q_{i j}=\frac{\pi_{i}\left(1-\hat{p}_{i j}\right) \hat{f}_{i j}\left(x_{j}\right)}{\pi_{1}\left(1-\hat{p}_{1 j}\right) \hat{f}_{1 j}\left(x_{j}\right)+\cdots+\pi_{n_{t}}\left(1-\hat{p}_{n_{t} j}\right) \hat{f}_{n_{t} j}\left(x_{j}\right)}
$$

where $\hat{f}_{i j}$ is the estimated probability mass/density function of $F_{i j}^{+}$. When $F_{i j}^{+}$is a continuous distribution, $\hat{f}_{i j}$ is a continuous density function, so 
$q_{i j}$ in (4) is not a real probability but we still call it a posterior probability here for the purpose of classifying the cell. If we only use the expression level of the $j$ th gene, we would assign the cell to type- $i^{*}$ where

$$
q_{i^{*} j}=\max \left\{q_{1 j}, q_{2 j}, \ldots, q_{n_{t} j}\right\}
$$

by the rule of maximum posterior probability. This is a weak learner in the sense that it is based on the expression level of only one gene.

\subsection{Combiner functions and the strong learner scAnnotate}

With $n_{g}$ genes, we need to combine information in the resulting $n_{g}$ weak learners to obtain an overall classification of the cell based on all $n_{g}$ genes. To this end, we define a combiner function

$$
s_{i}=s\left(q_{i 1}, q_{i 2}, \ldots, q_{i, n_{g}}\right) \quad \text { for } i=1,2, \ldots, n_{t}
$$

to combine the posterior probabilities for type- $i$ from all $n_{g}$ genes and classify the cell as type- $i^{*}$ where

$$
i^{*}=\underset{i}{\arg \max }\left\{s_{1}, s_{2}, \ldots, s_{n_{t}}\right\} .
$$

We call steps (1)-(7) mixture model based supervised classification of a cell. For convenience, we will refer to this method as scAnnotate.

\subsubsection{Example combiners}

We now give several examples of the combiner function (6). The first example is the voting score combiner function

$$
s_{i}=s_{A}\left(q_{i 1}, q_{i 2}, \ldots, q_{i, n_{g}}\right)=\sum_{j=1}^{n_{g}} I\left(q_{i j}\right)
$$

where $I\left(q_{i j}\right)=1$ if $q_{i j}=\max \left\{q_{1 j}, q_{2 j}, \ldots, q_{n_{t} j}\right\}$ and $I\left(q_{i j}\right)=$ 0 otherwise. This combiner function essentially counts the number of genes $s_{i}$ that give the cell a type- $i$ classification by the rule of maximum posterior probability shown in (5). With this combiner function, by (5) and (7), scAnnotate classifies a cell as a type- $i$ cell if it is most frequently classified/voted as a type- $i$ cell by the $n_{g}$ weak learners.

Another example is the weighted average of the posterior probabilities for type- $i$

$$
s_{i}=s_{B}\left(q_{i 1}, q_{i 2}, \ldots, q_{i, n_{g}}\right)=\sum_{j=1}^{n_{g}} w_{j} q_{i j}
$$

where the weight $w_{j} \geq 0$ and it represents the importance of the $j$ th gene. Such an importance may, for example, be a quantitative measure of how accurate the classification is when only $q_{1 j}, q_{2 j}, \ldots, q_{n_{t j}}$ are used to classify cells in the test data through (5). We may also apply the weights to define a weighted version of the voting score combiner,

$$
s_{i}^{\prime}=s_{A}^{\prime}\left(q_{i 1}, q_{i 2}, \ldots, q_{i, n_{g}}\right)=\sum_{j=1}^{n_{g}} w_{j} I\left(q_{i j}\right) .
$$

The last combiner example we include here is the weighted sum of log-transformed $q_{i j}$-scores defined as

$$
s_{i}=s_{C}\left(q_{i 1}, q_{i 2}, \ldots, q_{i, n_{g}}\right)=\sum_{j=1}^{n_{g}} w_{j} \log \left(q_{i j}\right),
$$

which is equivalent to the product $\Pi_{j=1}^{n_{g}} q_{i j}^{w_{j}}$. When we use uniform prior $\pi_{i}=P($ type- $i)=1 / n_{t}$ and equal weights $w_{j}=1$ with combiner $s_{C}$, it is equivalent to $\prod_{j=1}^{n_{g}} q_{i j} \propto \prod_{j=1}^{n_{g}} P\left(X_{j}=x_{j} \mid\right.$ type $\left.=i\right)$, and our ensemble learning reduces to the well-known Naive Bayes Classifier
(Rish et al., 2001). To see this, the Naive Bayes Classifier uses posterior probability,

$$
\begin{aligned}
s_{i}^{n b} & =P\left(\text { type }=i \mid X_{1}, \ldots, X_{n_{g}}\right) \\
& =\frac{P\left(X_{1}, \ldots, X_{n_{g}} \mid \text { type }=i\right) P(\text { type }=i)}{P\left(X_{1}, \ldots, X_{n_{g}}\right)} \\
& =\frac{\prod_{j=1}^{n_{g}} P\left(X_{j}=x_{j} \mid \text { type }=i\right) P(\text { type }=i)}{P\left(X_{1}, \ldots, X_{n_{g}}\right)} \\
& \propto \prod_{j=1}^{n_{g}} P\left(X_{j}=x_{j} \mid \text { type }=i\right),
\end{aligned}
$$

which is equivalent to using $\Pi_{j=1}^{n_{g}} q_{i j}$ or $s_{C}$ with uniform prior and equal weights. We investigated the combiners given above using multiple real scRNA-seq datasets and empirically found the combiner $s_{C}$ works best.

\subsubsection{Training the combiner}

The combiners involve the weights $w_{j}$, which need to be decided. We assign their values using two different approaches according to the sample size of the training data.

When the sample size is small, we do not have enough data to estimate parameters of all $F_{i j}$ and at the same time train a model to learn the weights $w_{j}$ for the combiner. In this case, we use all training cells to estimate the parameters of $F_{i j}$, and assume equal weights $w_{j}=1$ for all $j$.

When the training sample is large enough (e.g. about 600 observations per cell type and no less than 20 cells of any given type), we randomly split training data into two parts. The weights $w_{j}$ are learned via the following five steps. (1) We use $20 \%$ of the cells to estimate the parameters of the $F_{i j}$. (2) We use the estimated distributions to calculate $q_{i j}$ scores of the remaining $80 \%$ of cells. (3) Using the Wilcoxon Rank-Sum test, we filter out genes whose $q_{i j}$ scores are not highly associated with cell type labels, i.e. retain the top genes with smallest $p$-values. (4) Using these $80 \%$ cells' $q_{i j}$ scores as predictors and their corresponding cell types as outcomes, we train an Elastic Net model (Zou and Hastie, 2005) to learn the weights $w_{j}$. Note, to reduce the number of predictors, we apply PCA to the scores and use PC scores to replace the $q_{i j}$ scores. Since the Elastic Net model's result is a linear combination of PC scores, and the PC scores are linear combination of $q_{i j}$ scores, the final results are linear combinations of $q_{i j}$ scores. (5) To avoid sampling bias introduced by random data splitting, we repeat steps (1)-(4) for 10 times with different random splits, and use average weights learned from the 10 models as the final weights of combiner $s_{C}$.

\subsection{Implementation of scAnnotate}

Classification using scAnnotate depends on three key components: [I] the model for $F_{i j}^{+}$in (1), [II] the prior probabilities $\pi_{i}$ in (2), and [III] the combiner function in (6). For [I], we may use for example Negative Binomial distribution, Exponential distribution, lognormal distribution, or in situations where no good parametric models for positive expression levels are available, a nonparametric measure (see Section 2.7). Due to the usually huge number of combinations of $i$ (cell types) and $j$ (genes), it is not practical to model each individual $F_{i j}^{+}$separately, so we assume that distributions of all $F_{i j}^{+}$are of the same type, for example, all lognormal; and they can only differ in their parameter values. For [II], we use either a uniform prior or the observed proportions as discussed in Section 2.3. For [III], we may use one of the three combiner functions given above.

In real applications, we recommend using several combinations of the three components to build several classifiers with the training data, and then use test data to evaluate the performance of these classifiers using their $F_{1}$ scores to identify the optimal combination with the highest $F_{1}$ 
"output" — 2022/2/19 - 22:33 - page 5 - \#5

score. For real data, correct specifications of the components are unknown, so optimizing the combination by trying several combinations and choosing the best one protects scAnnotate from serious misspecifications of its components. For examples that we have tried, we found that the combination of lognormal distribution, uniform prior and combiner $s_{C}$ often has the best or second best performance. For simplicity of presentation, this combination is used in all examples in the next section.

Note that in cross-species and cross-platform studies, we need to apply batch effect removal techniques to preprocess the datasets and the processed datasets contain no zeros. For such processed datasets, the mixture model $F_{i j}$ in (1) for the $j$ th gene of a type- $i$ cell reduces to $F_{i j}^{+}$ as the proportion of zero $p_{i j}=0$. The mixture model $F_{j}$ in (2) remains unchanged. The implementation of scAnnotate also remains the same.

\subsection{Nonparametric depth measure for $F_{i j}^{+}$}

The true distribution of gene expression can be very complex. We may be unable to find, from the set of commonly used parametric distributions, a suitable one for modelling $F_{i j}^{+}$. The assumption that distributions for $F_{i j}^{+}$ of all genes are the same kind and that they can only differ in parameter values may also be too strong. To deal with these issues, when the sample size is large, we suggest using a nonparametric depth measure for $F_{i j}^{+}$ which is totally free of any parametric assumptions. Specifically, we may replace the estimated density function $\hat{f}_{i j}$ with a depth measure when computing the posterior probability in (4). We now illustrate this point with the use of the halfspace depth measure (Liu et al., 1999).

For a fixed gene of a fixed cell type, suppose there are $m$ non-zero expression data points from the training data set $x_{1}, x_{2}, \ldots, x_{m}$. Let $x^{*}$ be the expression level of that gene of the cell to be classified. The halfspace depth of $x^{*}$ measures how consistent $x^{*}$ is with the sample $x_{1}, x_{2}, \ldots, x_{m}$. It is defined as follows. First rank the $m+1$ observations in the augmented sample $x_{1}, x_{2}, \ldots, x_{m}, x^{*}$ and denote by $r\left(x^{*}\right)$ the rank of $x^{*}$. Then, the halfspace depth of $x^{*}, h\left(x^{*}\right)$, is given by

$$
h\left(x^{*}\right)=\frac{1}{m+1} \min \left\{r\left(x^{*}\right),(m+1)-r\left(x^{*}\right)+1\right\} .
$$

To see how $h\left(x^{*}\right)$ measures the consistency of $x^{*}$ with the data, when $x^{*}$ is the smallest or the largest of the augmented sample, $h\left(x^{*}\right)$ has its minimum value of $1 /(m+1)$, so a low $h\left(x^{*}\right)$ value indicates $x^{*}$ is not consistent with the data in that it is an extreme value. On the other hand, when $x^{*}$ is the median of the augmented sample, $r\left(x^{*}\right)=(m+1) / 2$ and $h\left(x^{*}\right)$ reaches its maximum value of $1 / 2$, so a large $h\left(x^{*}\right)$ value (close to $1 / 2$ ) indicate $x^{*}$ is consistent with the data. As such, it may be used to substitute $\hat{f}_{i j}$ in scAnnotate. Using this depth measure protects scAnnotate from severe misspecification of the model for $F_{i j}^{+}$. In simulation studies, scAnnotate based on the halfspace depth outperforms scAnnotate with severely misspecified $F_{i j}^{+}$in terms of accuracy. On the other hand, the depth measure requires the number of non-zero observations at all genes to be large (otherwise, the depth measure is too discrete to be a useful replacement for $\hat{f}_{i j}$ ) and is more computationally intensive.

\section{Results}

To evaluate the performance of scAnnotate, we conduct a benchmark study to compare it against nine other scRNA-seq annotation methods based on supervised machine learning algorithms, including scID (Boufea et al., 2020), scClassify (Lin et al., 2020), SingleCellNet (Tan and Cahan, 2019), scPred (Alquicira-Hernandez et al., 2019), CaSTLe (Lieberman et al., 2018), SingleR (Aran et al., 2019), CHETAH (de Kanter et al., 2019), scmapCluster and scmapCell (Kiselev et al., 2018). The parameters of these methods are chosen according to the suggestions in their vignettes or the software default settings; we note that all of the benchmarked methods have a fully automated data-driven approach without requiring previous biological knowledge. In our experiments, all models are learned on training data and then applied to annotate cells in test data. Prior to performing classification, we remove all cells whose cell types do not appear in both the training and test data. To evaluate the classification performance of the benchmarked methods, we compare the predicted labels of the test data with the corresponding true labels. Following the evaluation rule of other annotation method papers (Lieberman et al., 2018; Alquicira-Hernandez et al., 2019; Lin et al., 2020; Zhao et al., 2020), we use accuracy as our performance criteria. Accuracy in this study is defined as the percentage of correctly annotated cells.

We conduct the benchmark study under three situations according to the relationship between training and test data. (1) Training and test data are from the same platform (i.e. obtained from the same sequencing method) and the same species. (2) Training and test data are from different platforms. (3) Training and test data are from different species, human versus mouse.

\subsection{Datasets and preprocessing}

Table 1 summarizes the eleven publicly available scRNA-seq datasets used in our benchmark study. These data have been used to illustrate the annotation performance by nine competitor methods that we compare scAnnotate with in this section.

The human Peripheral Blood Mononuclear Cells (PBMC) scRNAseq data collection was downloaded from the SeuratData package (Hao et al., 2021) with dataset name "pbmcsca" (Ding et al., 2019) and consists of seven datasets that were sequenced using seven different methods: 10x Chromium (v2), 10x Chromium (v3), Drop-seq, Seq-Well, inDrops, Smart-seq2, and Cel-seq2. For cross-platform cell annotation, we removed all cells labelled as "Unassigned". Each dataset was then used as training data and all other datasets as test data. This gave us $7 * 6=42$ distinct pairs of cross-platform datasets.

Mouse and human pancreatic scRNA-seq data was downloaded from the National Center for Biotechnology Information (NCBI) Gene Expression Omnibus (GEO) for GSE84133 (Baron et al., 2016). Additional human pancreatic scRNA-seq data was downloaded from the SeuratData package (Hao et al., 2021) with dataset name "panc8" (Lab, 2019). We chose three datasets produced across different technologies: CelSeq (GSE81076) (Grün et al., 2016), indrop (GSE84133) (Baron et al., 2016) and SMART-Seq2 (E-MTAB-5061) (Segerstolpe et al., 2016). In preparation for cross-species cell annotation, we converted the human gene symbols to mouse ortholog gene symbols using the ortholog table provided by SingleCellNet (Tan and Cahan, 2019). The analysis was limited to common genes between the training and test data. We first used the Baron et al. (2016) mouse pancreatic data as training data and all human pancreatic datasets as test data. We then switched the trainingtesting order and used the human pancreatic data as training data in order to classify the mouse pancreatic data. This gave us $3 * 2=6$ distinct pairs of cross-species datasets.

The Baron et al. (2016) human dataset (GSE84133) was also used for intra-dataset evaluation. We applied stratified (by cell types) sampling to select $80 \%$ of the sample as training data and set the remaining $20 \%$ of the sample as test data. To investigate the variance in performance evaluation caused by sampling bias, we repeated this experiment ten times with different random data splitting.

The Seurat (version 4.0.5) (Hao et al., 2021) package was used for normalization and feature selection on all raw count matrices. The datasets were normalized using the NormalizeData function with the "LogNormalize" method and a scale factor of 10,000. After modeling the mean-variance relationship with the FindVariableFeature function within 


\begin{tabular}{lllllll}
\hline \multicolumn{1}{l}{ Dataset } & Description & Protocol & Cells & Genes & Cell types & Reference \\
\hline PBMC.10Xv2 & PBMC & 10X Chromium (v2) & 9806 & 33694 & 9 & Ding et al. $(2019)$ \\
PBMC.10Xv3 & PBMC & 10X Chromium (v3) & 3222 & 33694 & 8 & Ding et al. $(2019)$ \\
PBMC.DS & PBMC & Drop-seq & 6584 & 33694 & 9 & Ding et al. $(2019)$ \\
PBMC.SW & PBMC & Seq-Well & 3727 & 33694 & 7 & Ding et al. $(2019)$ \\
PBMC.ID & PBMC & inDrops & 6584 & 33694 & 9 & Ding et al. $(2019)$ \\
PBMC.SS & PBMC & Smart-seq2 & 526 & 33694 & 7 & Ding et al. $(2019)$ \\
PBMC.CS & PBMC & Cel-seq2 & 526 & 33694 & 7 & Ding et al. $(2019)$ \\
Baron (Mouse) & Mouse pancreas & inDrops & 1886 & 14878 & 13 & Baron et al. $(2016)$ \\
Baron (Human) & Human pancreas & inDrops & 8569 & 34363 & 13 & Baron et al. $(2016)$ \\
Segerstolpe & Human pancreas & Smart-seq2 & 2394 & 34363 & 13 & Segerstolpe et al. $(2016)$ \\
Muraro & Human pancreas & CelSeq & 1004 & 34363 & 13 & Grün et al. (2016)
\end{tabular}

Table 1. Overview of the datasets used in this study

"vst" methods, we selected the top 2000 highly variable genes and only used this selection going forward.

\section{2 scAnnotate can accurately classify human pancreatic cells using similar training and test scRNA-seq data}

When we randomly split the Baron et al. (2016) human pancreatic dataset (GSE84133) into training and test data as described in 3.1, scAnnotate (assuming $F_{i j}^{+}$follows a log-normal distribution) classified fourteen pancreatic cell types with a high overall prediction accuracy range of $96.79 \%$ to $97.90 \%$. The median prediction accuracy over the ten rounds of classification was $97.34 \%$, and the mean prediction accuracy was $97.39 \%$ Specifically, scAnnotate classified acinar, activated stellate, alpha, beta, delta, ductal, endothelial, epsilon, gamma, macrophage, mast, quiescent stellate, Schwann, and T cells with mean F1 scores 0.9618, 0.9504, $0.9910,0.9876,0.9481,0.9679,0.9839,0.4498,0.9699,0.9623,0.9636$, $0.9053,0.8357$, and 0.7205 , respectively. We note that the lower mean F1 scores resulted from classifying epsilon, Schwann, and T cells. These three cell types were rare in this dataset; out of the total 17,134 observed cells, epsilon, Schwann, and T cells had only 36,26 , and 14 respective observations. Figure 3 shows the accuracy comparison of scAnnotate and competitor methods, including the discriminative models scClassify, SingleCellNet, scPred, CaSTLe, SingleR, scmapCluster, scmapCell, CHETAH, and the generative model scID. Multiple methods showed high classification accuracies when training and test data were similar enough. Among these top performers, scAnnotate ranked third.

\section{3 scAnnotate can accurately classify PBMCs across different ScRNA-seq data generating platforms}

We used the PBMC datasets (Ding et al., 2019; Hao et al., 2021) provided by the Seurat Package to evaluate annotation methods' classification performances when training and test data are obtained from different scRNA-seq generating platforms. This data consists of scRNA-seq data generated using 7 different platforms as listed in Table 1, which leads to 42 pairs of cross-platform training and test data. We applied scAnnotate and competitor methods to each of these 42 data pairs and calculated their prediction accuracies

scAnnotate (assuming $F_{i j}^{+}$follows a log-normal distribution) had the highest median accuracy $84.70 \%$ and the second highest mean accuracy $81.22 \%$, and an overall prediction accuracy range of $43.82 \%$ to $92.77 \%$. Of the nine other benchmarked methods, only scPred had a comparably consistent cross-platform performance and had a similar box position in Figure 4A (with median and mean prediction accuracy $84.32 \%$ and $81.88 \%$, respectively). scAnnotate is thus a consistent and accurate contender for automated, cross-platform annotation of PBMCs. When we

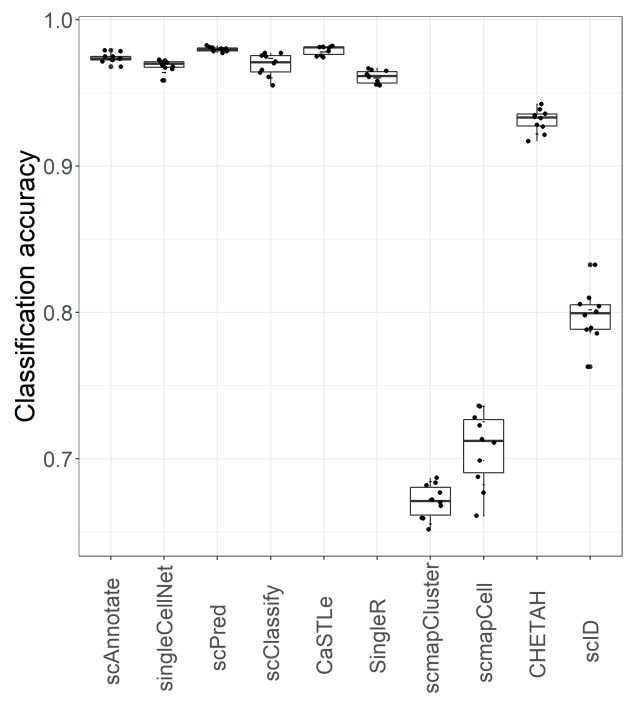

Fig. 3. Within-study classification performance of scAnnotate on the Baron et al. (2016) human pancreatic scRNA-seq dataset (GSE84133). The boxplots show the classification accuracies of scAnnotate and nine competitor methods in ten experiments on different random splits of the original data ( $80 \%$ training and $20 \%$ testing). Multiple methods show high accuracies when training and test data are similar enough. Among these top performers, scAnnotate ranks 3rd with a prediction accuracy range of $96.79 \%$ to $97.90 \%$ on fourteen pancreatic cell types.

look into individual settings, scAnnotate is among the top-ranked most accurate methods in most of the 42 cross-platforms settings (Figure 4B). When scAnnotate is not the best method, its accuracy is often not much lower than that of the winners.

\section{4 scAnnotate can accurately classify human pancreatic cells using scRNA-seq pancreatic data collected from mice and vice versa}

We first trained scAnnotate (assuming that $F_{i j}^{+}$follows a log-normal distribution) and nine other cell annotation methods on the Baron et al. (2016) mouse pancreatic dataset and predicted ten pancreatic cell types for the human datasets provided by Baron et al. (2016), Segerstolpe et al. (2016), and Grün et al. (2016). On these three combinations of mouse-human datasets, scAnnotate classified stellate, alpha, beta, delta, ductal, endothelial, gamma, macrophage, quiescent stellate, and Schwann cells with overall prediction accuracies of $89.55 \%, 71.84 \%$, and $71.41 \%$, respectively. As shown in Figure 5, scAnnotate consistently ranks in the top 


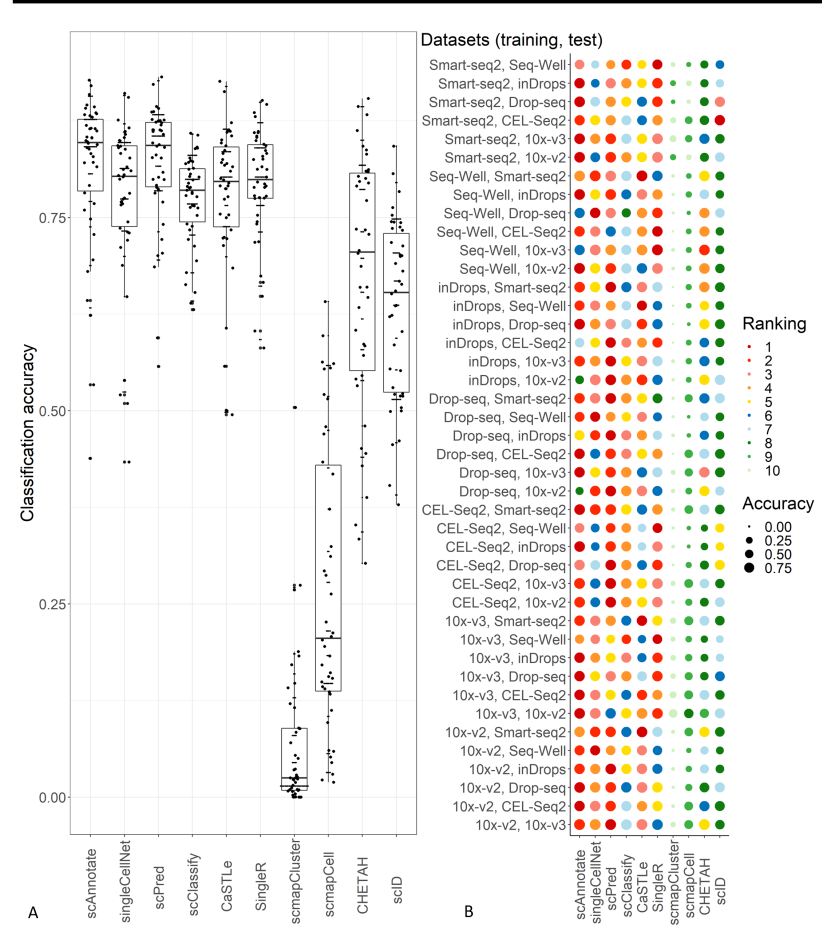

Fig. 4. Classification performance of scAnnotate on 42 combinations of cross-platform peripheral blood mononuclear cell (PBMC) scRNA-seq datasets, as provided by Ding et al. (2019). Panel (A) summarizes the overall comparison of methods under all settings. Each boxplot is constructed using the accuracy values of the method in 42 settings. The actual accuracy values are shown as dots together with their corresponding boxes. scAnnotate and scPred have the highest boxes indicating they are the two best methods in the overall comparison of 42 settings. Panel (B) shows the comparison results of each individual setting. Each row represents one setting of training and test data combination. Each circle represents the performance of one method. The colours of circles represent methods' ranks, and the sizes of circles represent their corresponding accuracies. scAnnotate consistently ranks in the top four most accurate methods in all settings. When scAnnotate is not the best method, its accuracy is not much lower than winners. Of the ten benchmarked methods, scAnnotate has the second highest mean accuracy $(81.22 \%)$ and the highest median accuracy $(84.70 \%)$.

four best performing methods for mouse to human cross-species pancreatic cell annotation. To further investigate the performance of scAnnotate on cross-species data, we then trained scAnnotate on the human pancreatic datasets listed above and classified the same ten pancreatic cell types for the Baron et al. (2016) mouse dataset. When trained on the Baron et al. (2016), Grün et al. (2016) and Segerstolpe et al. (2016) human datasets, scAnnotate had prediction accuracies of $89.15 \%, 73.46 \%$, and $47.02 \%$, respectively. Notably, scAnnotate had the highest classification accuracy of all ten benchmarked methods when trained and tested on the Baron et al. (2016) mouse and human data combination (Figure 5B); this result is promising, as the goal of cross-species cell annotation is often to classify human samples based on mouse samples. scAnnotate also had the second best performance of all ten benchmarked methods when trained on the Grün et al. (2016) human dataset and tested on the Baron et al. (2016) mouse dataset, which further demonstrates the potential of scAnnotate for cross-species cell annotation. Additionally, we note that the box position of scAnnotate's cross-species classification accuracies in Figure $5 \mathrm{~A}$ is relatively high compared to that of competitor methods. Thus, of the ten methods tested, scAnnotate is an accurate and reasonably consistent contender for pancreatic cross-species cell annotation.

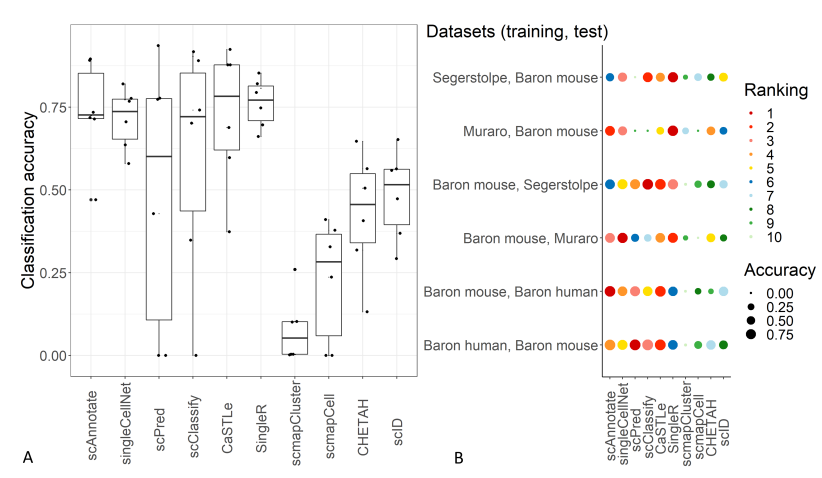

Fig. 5. Cross-species classification performance of scAnnotate on six combinations of mouse and human pancreatic scRNA-seq datasets. The mouse dataset is provided by Baron et al. (2016) (denoted 'Baron mouse'). The human datasets are provided by Baron et al. (2016) (denoted 'Baron human'), Grün et al. (2016) (denoted 'Muraro') and Segerstolpe et al. (2016) (denoted 'Segerstolpe'). Panel (A) summarizes the overall comparison of methods under all settings. Each scatter plot is constructed using the accuracy values of the method in the 6 cross-species settings. Panel (B) shows the comparison results of each individual setting. Each row represents one setting of training and test data combination. Each circle represents the performance of one method. The colours of circles represen methods' ranks, and the sizes of circles represent their corresponding accuracies. On any given dataset, a prediction accuracy of zero is assigned to methods that threw errors and failed to run. scAnnotate consistently ranks in the top four most accurate methods for the settings where training is done on mouse data and testing is done on human data. When scAnnotate is not the best method, its accuracy is not much lower than winners. The variation in accuracies for scAnnotate is comparable to that of the best methods, such as CaSTLe.

\section{Discussion}

scAnnotate is an analysis framework that consists of three major components. Users can change the components according to their own needs. First, we use two available softwares as a batch effect removal step: Harmony for small sample data, and Seurat for large sample data. Many batch effect removal methods can be used to replace them, such as the methods compared in the benchmark study by Tran et al. (2020). Second, an ElasticNet model is used to learn a combiner function. Users can choose whatever supervised machine learning models to replace it. For example, when the sample size is huge, we could train the combiner function using XGBoost (Chen and Guestrin, 2016), which is faster and much more precise than Elastic Net in this situation. Third, the distributions used in weak learners can also be changed. We will discuss this next.

After investigating many candidate distributions used in weak learners, we recommend using the lognormal distribution as the non-dropout component of our mixture model. Besides lognormal, we added two alternative distributions in our software for particular usages. The first alternative distribution is the exponential distribution. This distribution has only one parameter, and hence is most suitable for rare cell type detection with small and unbalanced sample sizes. The second alternative is the nonparametric distribution estimated using a depth function approach, used for extra-large sample size problems. We believe that all distributions oversimplify the complex truth of gene expression. Hence, a nonparametric approach can better estimate the complex true distribution when the sample size is big enough. We believe that this alternative will be more useful in the future as the sample size of scRNA-seq data continues to grow rapidly over the years.

Differential expression analysis uses genes one at a time; most DE methods therefore focus on modelling gene expression distribution to best utilize key features of genomic data. In contrast, annotation analysis needs to use all genes to make decisions. Most cell annotation methods do not model the distribution of genomic data, since it is hard to model the joint distribution of many genes. We address this challenge using an ensemble 
approach. We build classifiers using each gene separately and ensemble them using a combiner. This approach has three advantages in computing. First, by modelling each gene separately, the number of parameters in our model is linear in the number of genes, which successfully avoids the curse of dimensionality. Second, the estimation of distribution parameters in training data and the evaluation of posterior probability in test data often have close formed formulas, rather than the expensive iterative approximation used by many other types of classifiers. Third, when necessary, such calculations for tens of thousands of genes can be done in parallel to further reduce computing time.

While scAnnotate is developed as a stand-alone tool for cell annotation, it can also be used together with other methods. Besides ensemble weak learners of individual genes, we can also ensemble scAnnotate with many other annotation methods to build a 'mega' ensemble model. When computing time is not an issue, we believe that the mega model most likely outperforms all individual annotation methods. scAnnotate should be a key player in the mega model, since it is unique among the methods in the literature. It is the only method that explicitly models dropout (a critical characteristic of scRNA-seq), and is one of only two existing generative classifiers. Hence, we believe our method can provide complementary information to other cell annotation methods and play a critical role in the mega ensemble model of all methods.

In conclusion, we introduce scAnnotate, a streamlined process for scRNA-seq data analysis that includes data preprocessing and cell annotation. It is an entirely data-driven automated method that requires no biological knowledge and subjective human decisions. We simultaneously model genes' dropout proportions and expression levels via a twocomponent mixture model. We use an ensemble machine learning approach to address the curse of high-dimensionality. We build one weak classifier using each gene, and use a combiner function to integrate al weak classifiers into a single strong classifier. Using multiple real scRNAseq benchmark data sets, we show that scAnnotate accurately identifies cell types when training and test data are from (1) the same platform and species, (2) different scRNA-seq generating platforms, and (3) different species (specifically, mouse to human). Compared with other supervised learning methods, scAnnotate provides top-tier classification performance.

\section{Acknowledgements}

This research was enabled in part by support provided by WestGrid (www.westgrid.ca) and Compute Canada (www.computecanada.ca).

\section{Funding}

This work was supported by the Natural Sciences and Engineering Research Council Discovery Grants (XZ, XJ, Grant No. RGPIN-201704722) and (MT, Grant No. RGPIN-2016-03804), the Canada Research Chair (XZ, Grant No. 950-231363), Genome BC Sector Innovation Program (XZ, DT, KB).

\section{References}

Alquicira-Hernandez, J. et al. (2019). scpred: accurate supervised method for cell-type classification from single-cell rna-seq data. Genome biology, 20(1), 1-17.

Andrews, T. S. and Hemberg, M. (2018). False signals induced by singlecell imputation. F1000Research, 7.

Aran, D. et al. (2019). Reference-based analysis of lung singlecell sequencing reveals a transitional profibrotic macrophage. Nature immunology, 20(2), 163-172.
Artegiani, B. et al. (2017). A single-cell rna sequencing study reveals cellular and molecular dynamics of the hippocampal neurogenic niche. Cell reports, 21(11), 3271-3284.

Baron, M. et al. (2016). A single-cell transcriptomic map of the human and mouse pancreas reveals inter-and intra-cell population structure. Cell systems, 3(4), 346-360.

Boufea, K. et al. (2020). scid uses discriminant analysis to identify transcriptionally equivalent cell types across single-cell rna-seq data with batch effect. IScience, 23(3), 100914.

Chen, H. et al. (2019). Revolutionizing immunology with single-cell rna sequencing. Cellular \& molecular immunology, 16(3), 242-249.

Chen, T. and Guestrin, C. (2016). Xgboost: A scalable tree boosting system. In Proceedings of the 22nd acm sigkdd international conference on knowledge discovery and data mining, pages 785-794.

de Kanter, J. K. et al. (2019). Chetah: a selective, hierarchical cell type identification method for single-cell rna sequencing. Nucleic acids research, 47(16), e95-e95.

Diaz-Mejia, J. J. et al. (2019). Evaluation of methods to assign cell type labels to cell clusters from single-cell rna-sequencing data. F1000Research, 8.

Ding, J. et al. (2019). Systematic comparative analysis of single cell rna-sequencing methods. bioRxiv.

Finak, G. et al. (2015). Mast: a flexible statistical framework for assessing transcriptional changes and characterizing heterogeneity in single-cell rna sequencing data. Genome biology, 16(1), 1-13.

Gong, W. et al. (2018). Drimpute: imputing dropout events in single cell rna sequencing data. BMC bioinformatics, 19(1), 1-10.

Gown, A. M. (2008). Current issues in ER and HER2 testing by IHC in breast cancer. Modern pathology, 21(2), S8-S15.

Grün, D. et al. (2016). De novo prediction of stem cell identity using single-cell transcriptome data. Cell stem cell, 19(2), 266-277.

Hao, Y. et al. (2021). Integrated analysis of multimodal single-cell data. Cell.

Hicks, S. C. et al. (2018). Missing data and technical variability in singlecell rna-sequencing experiments. Biostatistics, 19(4), 562-578.

Huang, M. et al. (2018). Saver: gene expression recovery for single-cell rna sequencing. Nature methods, 15(7), 539-542.

Kharchenko, P. V. et al. (2014). Bayesian approach to single-cell differential expression analysis. Nature methods, 11(7), 740-742.

Kiselev, V. Y. et al. (2018). scmap: projection of single-cell rna-seq data across data sets. Nature methods, 15(5), 359-362.

Korsunsky, I. et al. (2018). Fast, sensitive, and flexible integration of single cell data with harmony. bioRxiv.

Lab, S. (2019). panc8.SeuratData: Eight Pancreas Datasets Across Five Technologies. R package version 3.0.2.

Leach, M. et al. (2013). Limitations, chapter 3, pages 20-30. John Wiley \& Sons, Ltd.

Li, W. V. and Li, J. J. (2018). An accurate and robust imputation method scimpute for single-cell rna-seq data. Nature communications, 9(1), 1-9. Lieberman, Y. et al. (2018). Castle-classification of single cells by transfer learning: harnessing the power of publicly available single cell rna sequencing experiments to annotate new experiments. PloS one, 13(10), $\mathrm{e} 0205499$.

Lin, Y. et al. (2020). scclassify: sample size estimation and multiscale classification of cells using single and multiple reference. Molecular systems biology, 16(6), e9389.

Liu, R. Y. et al. (1999). Multivariate analysis by data depth: Descriptive statistics, graphics and inference. The Annals of Statistics, 27(3), 783858 , with discussion.

Miao, Z. et al. (2018). Desingle for detecting three types of differential expression in single-cell rna-seq data. Bioinformatics, 34(18), 32233224. 
9(2), 207-213

Pasquini, G. et al. (2021). Automated methods for cell type annotation on scrna-seq data. Computational and Structural Biotechnology Journal.

Rish, I. et al. (2001). An empirical study of the naive bayes classifier. In IJCAI 2001 workshop on empirical methods in artificial intelligence, volume 3, pages 41-46.

Segerstolpe, Å. et al. (2016). Single-cell transcriptome profiling of human pancreatic islets in health and type 2 diabetes. Cell metabolism, 24(4), 593-607.

Soneson, C. and Robinson, M. D. (2018). Bias, robustness and scalability in single-cell differential expression analysis. Nature methods, 15(4), 255-261.

Tan, Y. and Cahan, P. (2019). Singlecellnet: a computational tool to classify single cell rna-seq data across platforms and across species. Cell systems,
Tang, F. et al. (2009). mrna-Seq whole-transcriptome analysis of a single cell. Nature methods, 6(5), 377-382.

Tran, H. T. N. et al. (2020). A benchmark of batch-effect correction methods for single-cell rna sequencing data. Genome biology, 21(1), $1-32$.

Zhao, X. et al. (2020). Evaluation of single-cell classifiers for single-cell rna sequencing data sets. Briefings in Bioinformatics, 21(5), 1581-1595. Zou, H. and Hastie, T. (2005). Regularization and variable selection via the elastic net. Journal of the royal statistical society: series B (statistical methodology), 67(2), 301-320. 